Conclusion We found ongoing potential STI/HIV transmission among MSM during the initial UK lockdown, despite a reduction in sexual activity, and potential inequalities in access to sexual healthcare. These findings will support public health planning to mitigate against health risks during and after the COVID-19 response.

\section{P237 DIAGNOSTIC AGREEMENT EVALUATION OF TREPONEMAL TEST FOR SYPHILIS TESTING}

\begin{abstract}
1,2S Vargas* ${ }^{3,4} \mathrm{~K}$ Konda, ${ }^{2}$ I Qquellon, ${ }^{2} \mathrm{~F}$ Vasquez, ${ }^{3} \mathrm{G}$ Calvo, ${ }^{3} \mathrm{M}$ Reyes, ${ }^{3} \mathrm{C}$ Caceres, ${ }^{4} \mathrm{~J}$ Klausner. ${ }^{1}$ School of Public Health and Administration, Universidad Peruana Cayetano Heredia, Lima, Perú; ' Laboratory of Sexual Health, Universidad Peruana Cayetano Heredia, Lima, Perú; ${ }^{3}$ Center for Interdisciplinary Studies in Sexuality, AIDS and Society, Universidad Peruana Cayetano Heredia, Lima, Perú; ${ }^{4}$ Division of Infectious Diseases, David Geffen School of Medicine, University of California Los Angeles, Los Angeles, USA
\end{abstract}

10.1136/sextrans-2021-sti.319

Background Laboratory diagnosis of syphilis infection requires treponemal and non-treponemal antibody tests. Treponemal pallidum (TP) antibody tests can be used for screening and confirmation, as part of a two-stage diagnostic algorithm. The diagnostic performance of treponemal tests should be assessed for potential implementation by local STI laboratories. We evaluated three treponemal antibody tests using serum samples from syphilis patients enrolled in a cohort study.

Methods The Picasso study is a cohort of participants with clinically diagnosed syphilis. Enrolment criteria required a positive rapid point-of-care TP test (Alere Determine syphilis, Abbott Inc, USA) followed by qualitative and semi-quantitative RPR test or clinical evidence of syphilis infection, AND additional confirmatory testing with TPPA (Serodia TPPA, Fujirebio Inc, USA) and TPHA (Syphilis TPHA liquid, Human Diagnostics, Germany). Three comparisons of interest were: TPPA vs rapid TP, TPPA vs TPHA and TPHA vs rapid TP. For each comparison, statistical analysis included positive percent agreement (PPA) and prevalence-adjusted and biasadjusted kappa (PABAK) correlation coefficient (i.e. a modification of Cohen's kappa when prevalence is too high). TPHA-indeterminate results were considered negative for analyses.

Results The Picasso study has enrolled 163 participants; all of them had a positive rapid TP test; $85 \%$ had an $\mathrm{RPR} \geq 1: 8$; 160 had a positive TPPA test, and 142 had a positive TPHA test. The PPA between TPPA and rapid TP was 98.0\% with a PABAK of 0.96 . For TPPA vs TPHA comparison, the PPA was $89.2 \%$ with a PABAK of 0.78 . The PPA between rapid TP and TPHA was $87.2 \%$ with a PABAK of 0.74 .

Conclusion We found a good concordance when comparing TPHA with either the TPPA or rapid syphilis test.

\section{P238 RIGHT TEST, RIGHT TIME: ENSURING TIMELY RENAL FUNCTION MONITORING IN CLIENTS TAKING PREP}

J Golden*, L Harryman. Unity Sexual Health, University Hospitals Bristol and Weston NHS Foundation Trust, Bristol, UK

\subsection{6/sextrans-2021-sti.320}

Background Clients receiving PrEP (HIV Pre-Exposure Prophylaxis) require renal function (estimated Glomerular Filtration Rate (eGFR)) monitoring as detailed in BASHH/BHIVA guidelines. Annual monitoring is required for individuals $<40$ with normal baseline eGFR and no risk factors for kidney disease. Our busy urban sexual health clinic lacked a structured system for eGFR monitoring, with concern clients were being tested unnecessarily or not at all.

Method A retrospective review was conducted of all clients under 40 years who received PrEP between June and November 2020. eGFR results and additional tests performed without clinical justification were extracted from records in the year preceding the issue of each PrEP prescription. The number of unnecessary repeat eGFRs within 12 months was recorded.

Results 199 clients were identified of whom 186 (93.5\%) had eGFR checked in the year prior to issuing PrEP. 13 clients $(6.5 \%)$ therefore had inadequate eGFR monitoring whilst continuing to take PrEP.

Of those tested, 55 (29.6\%) had eGFR re-checked within a year without clinical justification with a total of 69 unnecessary tests performed. This equates to $£ 345$ expenditure on tests, six hours of clinical time administering results and overinvestigation of clients with minor fluctuations in eGFR.

We implemented a new pathway for eGFR testing, including a visible alert on each client's record allowing clinical staff to immediately see when the eGFR was last checked, and when the next is due.

Conclusion This study identified both under- and over-testing of eGFR for clients taking PrEP. Whilst it is vital that eGFR testing is not missed, over-testing wastes clinical and financial resources which are at a premium in an era of budgetary constraints and reduced appointment availability due to COVID19. As the number of clients taking PrEP increases, it is important for all services to ensure robust and efficient methods of eGFR monitoring.

\section{P239 CLINICAL OUTCOMES OF SYPHILIS IN HIV-NEGATIVE AND HIV-POSITIVE MSM: OCCURRENCE OF REPEAT SYPHILIS EPISODES AND NON-TREPONEMAL SEROLOGY RESPONSES}

${ }^{1} \mathrm{R}$ Sprenger*, ${ }^{1,2} \mathrm{M}$ Schim-van der Loeff, ${ }^{1,2} \mathrm{H}$ de Vries. ${ }^{1}$ Public Health Service Amsterdam, Amsterdam, The Netherlands; ${ }^{2}$ Amsterdam UMC, Amsterdam, The Netherlands

\subsection{6/sextrans-2021-sti.321}

Objectives HIV-positive men who have sex with men (MSM) may be at a higher risk of repeat syphilis, have different clinical manifestations, and have a different serological response to treatment compared to HIV-negative MSM. The objective of this study was to assess whether HIV-negative and HIV-positive MSM with infectious syphilis (primary, secondary or early latent), differed in history of previous syphilis episodes, disease stage and non-treponemal titre of initial and repeat episodes, and the titre response 6 and 12 months after treatment. Furthermore, the determinants associated with an inadequate titre response after treatment were explored.

Methods This retrospective analysis used data of five longitudinal studies (4 cohorts and one RCT) conducted at a large sexually transmitted infection (STI) clinic in Amsterdam, the Netherlands. Participants were tested for syphilis and completed questionnaires on sexual risk behaviour every 3 or 6 months. We included data of participants with one or more syphilis diagnoses in 2014-2019. 